\title{
Méthode de comparaison d'images satellitaires pour la détection des changements en milieu forestier. Application aux monts de Lacaune (Tarn, France)
}

\author{
S Durrieu ${ }^{1}, M$ Deshayes 2 \\ 1 Inventaire forestier national, BP 1, 34970 Maurin; \\ 2 Laboratoire commun de télédétection CEMAGREF/ENGREF, \\ domaine de Lavalette, BP 5095, 34033 Montpellier cedex 1, France
}

(Reçu le 4 mai; accepté le 10 novembre 1993)

\begin{abstract}
Résumé - La télédétection satellitaire est, d'après certaines études, un outil adapté à la détection et à la cartographie des changements forestiers importants (coupes rases, incendies) sur de vastes territoires. Elle intéresse donc l'Inventaire forestier national français (IFN) dans une optique de mise à jour de sa carte des types de peuplements. Dans la présente étude nous avons cherché à détecter des changements plus subtils, tels les éclaircies. L'approche retenue consiste à normaliser des images satellitaires prises à des dates différentes et à les comparer ensuite pixel à pixel. La méthode de correction relative des effets atmosphériques proposée est basée sur les seules données image. Elle a été testée sur une zone de moyenne montagne, les monts de Lacaune (Tarn), à partir d'images Landsat TM prises à 1 et 2 ans d'intervalle (août 1988, 1990 et 1991). Les premiers résultats obtenus par comparaison des images après normalisation sont très satisfaisants en ce qui concerne la détection des coupes rases et des éclaircies. D'autres changements tels que dégagements dans des reboisements, travaux sur pistes, incendies sont aussi détectés. Ces résultats sont encourageants et il faut maintenant se préoccuper de la façon dont ils peuvent être cartographiés et présentés auprès des utilisateurs des données IFN.
\end{abstract}

cartographie forestière / télédétection satellitaire / détection des changements / prétraitements / correction radiométrique / éclaircies

Summary - A method of comparing satellite images to detect forest changes in an area of rugged terrain (southwestern France). According to the literature, satellite remote sensing is a tool adapted to the detection and mapping of major forest changes (clearcuts, burned areas) on vast territories. The French forest survey service, Inventaire Forestier National (IFN), is therefore interested in its use for updating its map of stand types. The present study attempts to detect small changes, mainly thinnings. The approach consists in normalizing satellite images taken at different dates and comparing them pixel by pixel. The proposed method of relative correction of atmospheric effects is based on image data only. It has been tested in a relief area, Monts de Lacaune (Tarn, southwestern France), and on Landsat TM images taken in August 1988, 1990 and 
1991. The initial results, obtained by image comparison after normalization, concerning clearcuts and thinnings are good. Other changes, such as cleaning, forest road works, and burned areas are also detected. These results are encouraging and the work now consists in actual mapping of the changes in a way which is suitable for users.

forest mapping / satellite remote sensing / change detection / preprocessing / radiometric correction / thinning

\section{INTRODUCTION}

En France, l'Inventaire forestier national (IFN) s'intéresse depuis quelques années déjà aux possibilités d'utiliser la télédétection satellitaire pour mener à bien certaines de ses missions (Bazire, 1987). L'IFN a été créé en 1958 pour procéder à l'inventaire permanent des ressources forestières afin de fournir les informations nécessaires pour décider de la politique forestière française. La méthodologie d'inventaire comprend une étape de cartographie précise de types de peuplements, définis pour ses besoins par I'IFN et caractérisés par l'essence dominante, le mode de conduite (qui détermine en partie la structure), des classes de volume... Pour chaque département ce travail est réalisé tous les 10 ans à partir de photographies aériennes au 1/17 000 ou au $1 / 25000$; le photo-interprète reporte sur le fond de carte topographique IGN au $1 / 25000$ (parfois au 1/50 000) les limites des types de peuplements (IFN, 1988). Cette cartographie ne semble pas réalisable avec les capteurs haute résolution spatiale des satellites actuels d'observation de la Terre (Strome et al, 1991 ; Peyron et al, 1988). Leurs résolutions spatiales et spectrales ne suffisent pas pour extraire toute l'information nécessaire à une cartographie aussi précise que celle de I'IFN. À partir des images satellitaires il est en effet possible de distinguer des grands groupes tels que feuillus, résineux, mélange feuillus/résineux, coupes rases, régénération et partois de déterminer quelques classes de densité (Leckie, 1989). L'utilisation d'informations extérieures, par exemple d'un modèle numé- rique de terrain pour déterminer les zones les plus probables de localisation d'une essence lorsqu'on connaît ses préférences écologiques, peut permettre d'affiner les résultats obtenus par classification (USDA, 1981 ; Franklin et al, 1986). Mais ces améliorations sont insuffisantes pour répondre aux besoins de l'IFN.

Cependant l'imagerie satellitaire paraît prometteuse pour fournir certaines informations demandées par les utilisateurs actuels et potentiels des données de l'IFN (Deshayes et al, 1990 ; Lepoutre et al, 1986). Des recherches sont donc menées, en partie dans le cadre du projet Spot et Forêts 2 (Deshayes et al, 1992), sur l'utilisation de ces images pour l'enrichissement et la mise à jour des cartes de peuplements de l'IFN.

L'enrichissement de l'information contenue sur la carte des types de peuplements repose sur le constat que, s'il n'est pas possible de retrouver la typologie IFN à partir d'images satellitaires, certaines unités cartographiées par le photo-interprète présentent une hétérogénéité radiométrique qui est porteuse d'information. ॥ est par exemple envisageable de préciser l'état du type "reboisements résineux", classe IFN qui comprend des peuplements de 0 à 40 ans qui peuvent être fermés ou non, suivant leur âge. Cependant sans correction des effets topographiques, qui induisent des différences de radiométries importantes entre versants à l'ombre et au soleil, il est impossible d'étendre systématiquement à toute l'image une relation (radiométrie/caractéristique du peuplement), constatée localement sur le terrain (Fontaine, 
1992). De nombreux auteurs se sont penchés sur ce problème difficile de correction des effets topographiques (Proy et Leprieur, 1986 ; Yang et Vidal, 1990) mais les résultats obtenus en utilisant les modèles proposés sont rarement entièrement satisfaisants (Lauret, 1991).

Les mises à jour intermédiaires de la carte des peuplements sont destinées à fournir aux utilisateurs une image actuelle de la forêt. Des recherches sont encore nécessaires dans ce domaine car, s'il est facile de détecter visuellement ou parfois de façon automatique des perturbations importantes (coupes rases, incendies, dégâts de tempête...), il est nécessaire d'améliorer les techniques pour la cartographie précise de changements plus subtils (coupes partielles par exemple) (Leckie, 1989).

Dans une optique de mise à jour de la carte des peuplements de l'IFN, nous avons fait le choix de comparer l'évolution radiométrique pixel à pixel entre 2 images prises à 2 dates différentes. Les principaux problèmes à résoudre sont alors :

- de rendre les images comparables entre elles;

- de déterminer un seuil de «changement» radiométrique ;

- d'arriver à prendre en compte des décalages qui persistent entre les images après corrections géométriques.

Dans cet article nous allons nous intéresser aux 2 premiers problèmes par la mise au point d'une méthode de normalisation relative basée sur les seules données images et par la définition d'un seuil de changement. La normalisation aura comme objectif principal, dans notre cas, de «neutraliser les effets atmosphériques".

Après avoir présenté la zone d'étude, nous décrirons la méthode proposée. Les résultats obtenus par application de cette méthode seront comparés à des informations de terrain afin de connaitre les types de changements détectables et, en ce qui concerne les coupes d'éclaircie, leur taux de détection.

\section{MATÉRIEL ET MÉTHODES}

\section{La zone d'étude}

L'étude a été réalisée sur une partie d'une région forestière diversifiée de moyenne montagne, les monts de Lacaune, située à l'est du département du Tarn. Les données disponibles sur cette zone sont :

- les cartes topographiques IGN au $1 / 25000$ et $1 / 50000$ correspondantes ;

- la carte des types de peuplement IFN réalisée à partir de photographies aériennes de 1988 ;

- un modèle numérique de terrain (MNT, avec altitudes, pentes et orientations) au pas de $20 \mathrm{~m}$;

- les images Landsat TM du 01/08/08, du 23/08/90 et du $26 / 08 / 91$;

- les photographies aériennes IRC au 1/17000 de I'IFN ;

- des informations sur l'évolution des peuplements fournies par I'ONF (Office national des forêts), le CRPF (Centre régional de la propriété forestière) et des visites de terrain.

Les principaux peuplements forestiers, suivant la nomenclature de I'IFN, se répartissent comme suit :

Futaie de hêtres:

$3,7 \%$

Futaie autres feuillus

(chênes, mélanges) :

$4,8 \%$

Total futaie feuillus :

Taillis de chênes :

$3,6 \%$

Taillis de hêtres :

$5,1 \%$

Autres taillis

(mélanges et châtaigniers) : $\quad 22,2 \%$

Total taillis feuillus

$30,9 \%$

Futaie + taillis feuillus

$3,9 \%$

Total feuillus

(futaie + taillis)

$43,3 \%$

Futaie de sapins ou d'épicéas : $\quad 2,0 \%$

Autre futaie de conifères

(mélanges, pins, douglas...) : $\quad 0,4 \%$

Total futaie résineux : 
Reboisement résineux en plein (<40 ans) :

$40,3 \%$

Reboisement résineux

en bandes ( $<40$ ans) :

Total reboisements :

$2,9 \%$

$43,2 \%$

Total résineux

(futaie + reboisements) :

Mélange feuillus résineux :

Boisements morcelés et lâches (feuillus et résineux) :

Sur la zone étudiée, l'altitude varie de 300 à $1260 \mathrm{~m}$. Les peuplements forestiers sont situés sur des pentes de $11^{\circ}$ en moyenne avec $5 \%$ de la surface forestière sur des pentes supérieures à $23^{\circ}$. Toutes les expositions sont bien représentées bien que l'exposition nord-ouest soit légèrement dominante. Même si l'on constate certaines tendances (reboisements résineux sur des pentes plus faibles, peuplements de hêtre plutôt sur les versants nord), on trouve chaque type de peuplement dans des positions topographiques variées.

La plupart des boisements résineux sont jeunes et les coupes rases sont encore rares. Les éclaircies d'amélioration sont généralement réalisées suivant le schéma classique : éclaircie systématique (1 rang sur 5 ) + sélective dans les rangs restants. Les très jeunes peuplements peuvent subir des dégagements. Pour les feuillus, les intensités des éclaircies d'amélioration sont très variables (de 17 à $52 \mathrm{~m} 3 /$ ha prélevés pour les éclaircies signalées par l'ONF). L'extraction des bois se fait le long de layons bien visibles dans le peuplement. Les boisements feuillus sont aussi soumis à des coupes de régénération, quelques coupes rases et des coupes affouagères. Des changements d'origine accidentelle ont parfois touché les peuplements de la région: incendies, chablis et volis, maladies.

\section{Méthode}

Nous cherchons à savoir si certaines de ces perturbations sont visibles sur les images satellitaires. Ces images sont tout d'abord corrigées géométriquement en se servant du MNT. On obtient ainsi des ortho-images superposables entre elles et aux différentes cartes. Elles vont ensuite subir des corrections radiométriques qui nécessitent de comprendre l'influence de cer- tains facteurs sur les valeurs enregistrées par le satellite.

\section{Facteurs de variation des comptes numériques entre images}

La donnée caractéristique de l'objet étudié est sa réflectance, $R$, rapport entre énergie réfléchie au niveau du sol et énergie incidente pour une longueur d'onde donnée. Au niveau du satellite le capteur mesure une luminance La, qui est enregistrée sous forme numérique et codée sur un octet (dans le cas, entre autres, des images Landsat TM et SPOT) ; les images sont donc constituées d'une matrice d'entiers variant de 0 à 255, souvent appelés comptes numériques. Ces luminances enregistrées par le satellite, permettent de calculer les réflectances apparentes, $\mathrm{Ra}$, au sommet de l'atmosphère.

Entre 2 images, 3 facteurs principaux sont susceptibles de modifier la mesure au niveau du capteur :

- l'état du couvert végétal : afin de limiter les différences d'état, difficiles à prendre en compte, les images ont été sélectionnées en été, quand la stabilité phénologique est grande, ce qui permet de supposer que le couvert a le même comportement spectral d'une année à l'autre ;

- la géométrie d'observation : la réflectance est directionnelle et dépend de l'angle d'incidence solaire $\theta_{s}$, de l'angle de prise de vue $\theta_{v}$ et de l'azimut relatif entre les directions d'observation et d'éclairement. En prenant des images à la même époque de l'année, l'angle d'incidence solaire sera considéré comme identique. De plus, nous avons choisi de travailler dans un premier temps avec des images Landsat TM qui sont toutes prises en visée verticale. Les effets liés aux conditions d'observation sont alors comparables pour un même pixel d'une date à l'autre ;

- les conditions atmosphériques vont jouer sur la réflectance apparente d'un objet car la composition très variable dans le temps de l'atmosphère (eau, aérosols...) modifie l'importance des phénomènes d'absorption et de diffusion par ses composants. Pour une cible de grande taille, la relation entre réflectance apparente et réflectance réelle de la cible est donnée par Deschamps et al (1981). Cette relation peut être approchée de façon satisfaisante par une loi linéaire $(\mathrm{Gu}, 1988)$ :

$$
R_{\mathrm{a}}=\operatorname{tg} \cdot\left[R_{\mathrm{atm}}+k \cdot R\right]
$$


avec $R_{a}$ : réflectance apparente; tg : facteur de transmission gazeuse (qui traduit le phénomène d'absorption par les gaz); $R_{\text {atm }}$ : réflectance atmosphérique; $R$ : réflectance réelle de la cible; $k$ : coefficient multiplicatif qui traduit les effets de transmission directs et diffus et les interactions multiples sol/atmosphère.

En ce qui concerne les 2 premiers facteurs, état de la végétation et conditions d'observation, nous nous sommes placés dans des conditions comparables. Seule l'influence du troisième, les conditions atmosphériques, a alors nécessité une correction.

\section{Correction relative des effets atmosphériques}

II s'agit donc de corriger les comptes numériques d'une image Ima2, prise à la date $\mathrm{t} 2$, pour les rendre comparables à ceux de l'image Ima1, prise à la date $\mathrm{t} 1$.

La correction idéale consisterait à utiliser les paramètres atmosphériques. Mais ces données sont rarement disponibles. Certaines méthodes les estiment en partant de l'hypothèse que la réflectance de lacs oligotrophes peut être assimilée à la réflectance atmosphérique, $R_{\text {atm }}$ (Poupard, 1991). Lopez Garcia (1987) présente une méthode intéressante mais qui nécessite un calage avec des mesures de radiométrie sur le terrain. Nous allons proposer une méthode de correction relative basée sur la recherche d'invariants entre images à partir des seules données image et applicable sous certaines conditions, auxquelles répond le milieu forestier étudié. Deux hypothèses vont nous permettre de réaliser cette transformation.

\section{Hypothèse 1}

Les perturbations atmosphériques sur la réflectance peuvent être approximées par une loi linéaire.

Cette hypothèse de linéarité a déjà été utilisée pour établir des relations entre valeurs radiométriques des images et réflectances mesurées au sol (Gu, 1988 ; Lopez Garcia, 1987). Elle va nous permettre, pour les pixels inchangés, d'établir une relation linéaire entre comptes numériques de 2 images prises à des dates différentes.

À la date $t_{i}$ on a en effet les relations suivantes: - i) Passage de la réflectance apparente à la luminance mesurée par le capteur:

$$
\mathrm{L}_{\mathrm{i}}=\left[\cos \left(\theta \mathrm{s}_{\mathrm{i}}\right) \cdot \mathrm{Es}_{\mathrm{i}} / \pi\right] \cdot \mathrm{Ra}
$$

avec $L_{i}$ : luminance en $W \cdot m^{-2} \cdot \mu m^{-1} \cdot s r^{-1} ; \cos \left(\theta s_{i}\right)$ : angle d'incidence solaire; $E s_{i}$ : éclairement au sommet de l'atmosphère en $\mathrm{W} \cdot \mathrm{m}^{-2} \cdot \mu \mathrm{m}^{-1}$; Ra : réflectance apparente.

Soit, en utilisant la relation [1] :

$$
L_{i}=\operatorname{tgi} \cdot\left[\cos \left(\theta_{s i}\right) \cdot E_{s i} / \pi\right] \cdot\left[\operatorname{Ratm}_{i}+k_{i} R_{i}\right]
$$

- ii) Passage de la luminance mesurée par le capteur au compte numérique de l'image :

$$
\mathrm{CN}_{\mathrm{i}}=\mathrm{A} \cdot \mathrm{L}_{\mathrm{i}}+\mathrm{B}
$$

$A$ et $B$ étant obtenus à partir des coefficients de calibration du capteur.

- iii) Pour les peuplements inchangés entre les dates $\mathrm{t} 1$ et $\mathrm{t} 2$ on $\mathrm{a}$ :

$$
R_{1}=R_{2}=R
$$

On obtient finalement une relation linéaire, valable pour les pixels inchangés, entre comptes numériques de la date 1 et de la date 2 à partir de [3], [4] et [5] :

$$
\mathrm{CN}_{1}=\mathrm{a} \cdot \mathrm{CN}_{2}+\mathrm{b}
$$

où $\mathrm{a}>0$.

Cette équation est aussi l'équation de normalisation de $\mathrm{CN}_{2}$ par rapport à $\mathrm{CN}_{1}$ pour l'ensemble des pixels :

$$
\mathrm{CN}_{2} \text { normalisé }=\mathrm{CN}_{2 n}=\mathrm{a} \cdot \mathrm{CN}_{2}+\mathrm{b}
$$

(avec $\mathrm{CN}_{2 n}=\mathrm{CN}_{1}$ pour les pixels inchangés)

\section{Hypothèse 2}

En zone forestière, les changements n'affectent qu'un pourcentage limité des peuplements. La recherche de parcelles invariantes, qui vont nous servir au calcul des coefficients a et $b$ de la relation linéaire trouvée précédemment, va se faire en deux temps.

Première étape : on considère qu'il n'y a aucun changement sur la zone forestière. On peut alors écrire à partir de [6] :

$$
\begin{aligned}
& \mu_{\mathrm{f} 1}=\mathrm{a} \mu_{\mathrm{f} 2}+\mathrm{b} \quad \mathrm{a}=\sigma_{\mathrm{f} 1} / \sigma_{\mathrm{t} 2} \\
& ==>
\end{aligned}
$$

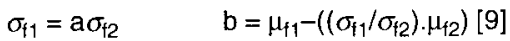




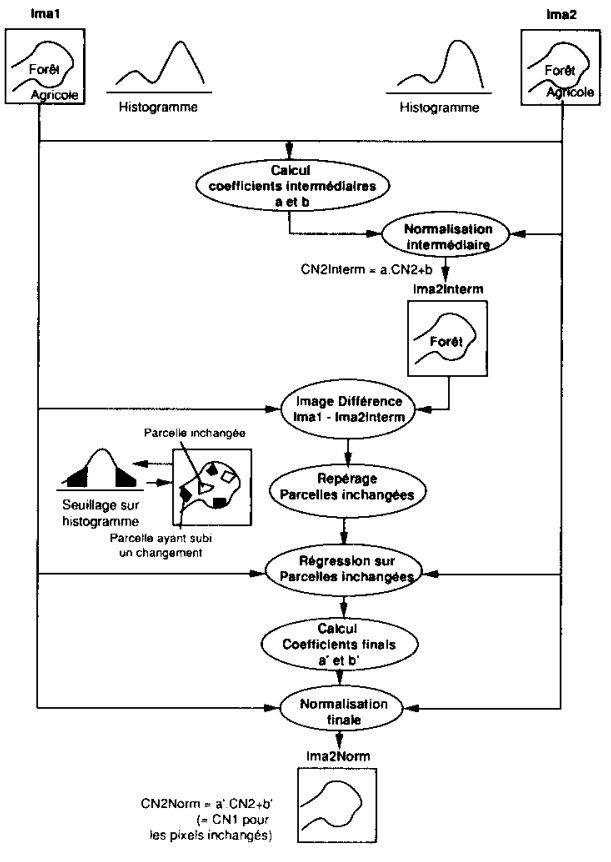

Fig 1. Schéma logique de la normalisation relative de 2 images.

avec $\mu_{\mathrm{f}}$ et $\sigma_{\mathrm{f}}$, moyenne et écart type des comptes numériques des zones forestières à la date ti $(i=$ $1,2)$, la carte de I'IFN permettant de déterminer ces zones forestières.

Deuxième étape : dans la réalité quelques changements ont eu lieu, qui peuvent modifier, par rapport à une situation sans perturbation, l'écart type et la moyenne des pixels forestiers et donc les valeurs a et b obtenues par [8] et [9]. II faut donc affiner le calcul des coefficients de normalisation (fig 1 ).

Pour cela on normalise l'image Ima2, prise à t2, par rapport à l'image Ima1, prise à t1, en utilisant les coefficients $a$ et $b$. On obtient l'image Ima2Interm. On calcule alors la différence, pixel à pixel, entre Ima2Interm et Ima1 par (CN2interm$\mathrm{CN} 1+128$ ) (l'ajout de la constante 128 permet d'éviter les valeurs négatives et de recentrer l'histogramme de l'image résultat entre les bornes 0 et 255). Si $a^{\prime}$ et $b^{\prime}$ sont les bons coefficients de normalisation, actuellement inconnus, on a :

$$
\begin{aligned}
& \mathrm{CN}_{2} \text { interm }=a \cdot \mathrm{CN}_{2}+b \\
& \mathrm{CN}_{2 n}=a^{\prime} \cdot \mathrm{CN}_{2}+b^{\prime} \\
& \left(\mathrm{CN}_{2 n}=\mathrm{CN}_{2} \text { normalisé }\right)
\end{aligned}
$$

D'où : $\mathrm{CN}_{2}$ Interm-CN $+128=\left(a / a^{\prime}-1\right) \cdot \mathrm{CN}_{2 n}+$ $\left(\mathrm{CN}_{2 n}-\mathrm{CN}_{1}\right)+b-a / a^{\prime} \cdot b^{\prime}+128$ 0) :

Soit pour les pixels inchangés $\left(\mathrm{CN}_{2 n}-\mathrm{CN}_{1}=\right.$

$\mathrm{CN}_{2}$ interm- $\mathrm{CN}_{1}+128$

$=\left(a / a^{\prime}-1\right) \cdot C N_{2 n}+b-a / a^{\prime} \cdot b^{\prime}+128$

Si la plupart des pixels n'ont pas changé, (a/a'1) sera proche de 0 et un grand nombre de points sur l'image des différences auront une valeur proche de 128 : l'histogramme de cette image présente effectivement un mode pour une valeur voisine de 128 . Les valeurs qui s'écartent beaucoup de ce mode sont liées :

- soit à un changement de radiométrie significatif ;

- soit à un problème de décalage résiduel après corrections géométriques en limite de peuplement ou sur des zones de forte texture.

On réalise donc un seuillage sur l'image différence (Ima2Interm-Ima1) afin de ne conserver qu'environ $60 \%$ de la population autour du mode de l'histogramme de cette image. En gardant seulement les pixels qui ont une valeur comprise entre $M-\sigma$ et $M+\sigma$ (avec $\sigma$ écart-type des comptes numériques de la différence d'images), on limite les risques de sélectionner des pixels qui ont subi un changement. Sur l'image seuillée, on va prendre une série de parcelles (une centaine) en essayant d'avoir une large gamme de valeurs pour les moyennes des comptes numériques de ces parcelles. On doit alors avoir, avec l'hypothèse de linéarité des effets atmosphériques, une relation linéaire entre les moyennes des parcelles entre 2 dates:

$$
\mu_{1}=a^{\prime} \mu_{2}+b^{\prime}
$$

Les coefficients a' et $b^{\prime}$, normalement différents de $a$ et $b$, sont calculés par régression linéaire sur l'ensemble des parcelles. La figure 2 présente un exemple de droite de régression obtenue à partir des moyennes radiométriques calculées sur les canaux TM4 (proche infra-rouge) de 1990 et 1991, pour un lot de parcelles inchangées.

L'utilisation de parcelles invariantes et non pas d'une série de points se justifie par : 
TM4 90-91

Parcelles inchangées

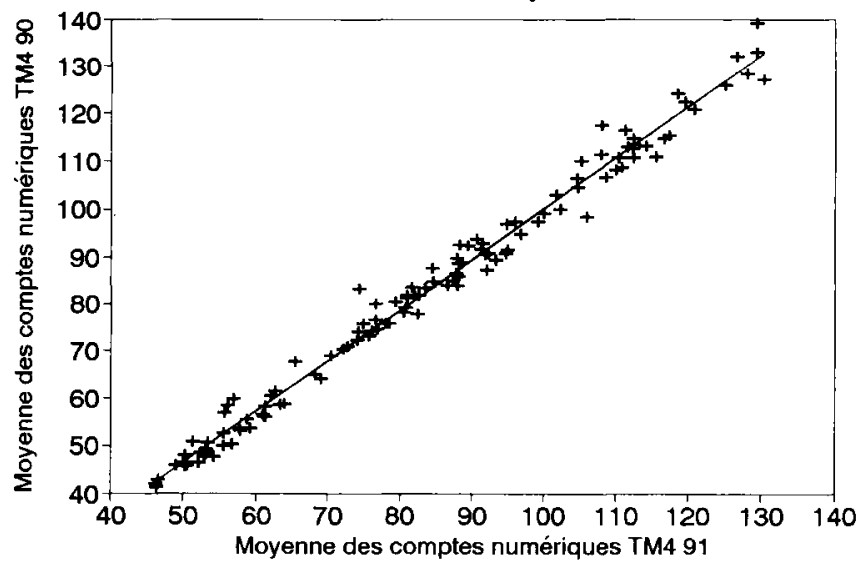

Fig 2. Moyennes radiométriques dans le proche-infra rouge (TM4) en 1990 et 1991 pour une série de parcelles inchangées et calcul de la droite de régression.

- les erreurs de localisation des pixels après correction géométrique qui sont, en erreur quadratique moyenne, inférieures au pixel au niveau des points d'amer mais qui peuvent atteindre localement jusqu'à 3 pixels dans cette zone de relief ;

- les problèmes d'influence de l'environnement sur le calcul des réflectances : en effet, la réflectance d'une cible est influencée par la réflectance Re de son environnement. Pour prendre en compte cet effet, Richter (1990) propose une méthode simple qui, d'après ltten et al (1992), donne de bons résultats :

$$
\text { Rréel }=R+q(R-R m o y)
$$

avec $\mathrm{B}$ : réflectance calculée par (1) en supposant la cible grande (> $1 \mathrm{~km}^{2}$ ); Rmoy : réflectance moyenne calculée sur une fenêtre centrée sur la cible (fenêtre $9 \times 9$ pour des images Landsat TM); $\mathrm{q}:$ facteur représentant de l'environnement qui est une fonction des caractéristiques de l'atmosphère.

En prenant la moyenne sur une parcelle, l'équation [14] donne:

$$
\mu \text { Rréel }=\mu R+q(\mu R-\mu R \text { moy })
$$

Si la parcelle est suffisamment grande on a $\mu R \approx \mu R$ moy, d'où $\mu$ Réel $=\mu R$.

\section{Calcul du seuil de changement}

L'intérêt de la méthode proposée est de foumir, en plus des coefficients de normalisation, un outil statistique de décision sur la présence d'une évolution radiométrique significative ou non. En effet une région de confiance est associée à la droite de régression estimée à partir des parcelles inchangées. Ses limites vont nous permettre de définir un seuil à partir duquel on considère que les comptes numériques ont évolués entre $t 1$ et t2. Pour $\mathrm{CN}_{2}$ donné, l'erreur de prédiction de $\mathrm{CN}_{2 n}\left(\mathrm{CN}_{2}\right.$ normalisé) peut être décrite par la variance de l'erreur:

$\operatorname{var}\left(\mathrm{CN}_{2 n}-\mathrm{CN}_{2 n}\right)$

$=\sigma^{2}\left(1+1 / n+\left(\left(\mathrm{CN}_{2}-\mu \mathrm{CN}_{2}\right)^{2} / \Sigma\left(\left(\mathrm{CN}_{2}\right)_{i}-\mu \mathrm{CN}_{2}\right)^{2}\right)\right)$

L'intervalle de confiance de $\mathrm{CN}_{2 n}$ au seuil de confiance $\alpha=0,05$ est donné par :

$\mathrm{CN}_{2 n} \pm S=\mathrm{CN}_{2 n} \pm \operatorname{et}\left(\mathrm{CN}_{2 n}-\mathrm{CN}_{2 n}\right) \cdot \mathrm{t}(\mathrm{n}-2, \alpha / 2)$

avec et $=$ écart type et $t$ variable de Student. 
Ainsi, si la différence entre $\mathrm{CN}_{1}$ et $\mathrm{CN}_{2}$ normalisé par les coefficients $a^{\prime}$ et $b^{\prime}\left(\mathrm{CN}_{2 n}\right)$ est en dehors de l'intervalle $[-S ; S$, on considérera qu'il $y$ a changement de radiométrie entre $t 1$ et $t 2$ (fig 3).

\section{Analyse des changements}

L'évaluation de la méthode pour la détection des changements s'est faite dans 2 "sens" :

- d'une part, en allant des images vers le terrain pour vérifier si les endroits détectés comme ayant changé radiométriquement correspondent à un changement réel au niveau du peuplement ;

- d'autre part, en partant d'informations de terrain : les parcelles de gestion pour lesquelles I'Office national des forêts (ONF) avait répertorié une intervention (coupes rases et éclaircies) ont été relevées et reportées sur les images. Ces parcelles ont été analysées radiométriquement pour voir si elles présentaient toutes une évolution radiométrique significative.

\section{RÉSULTATS}

Une première analyse nous avait permis de mettre en évidence une redondance de l'in-

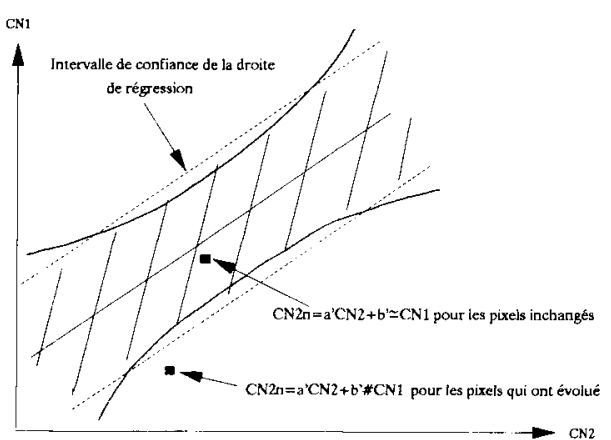

Fig 3. Droite de régression et intervalle de confiance : l'intervalle de confiance est limité par 2 branches d'hyperboles qui pourront ici, dans le cas d'effectifs suffisants, être approximées par 2 droites parallèles à la droite de régression, permettant ainsi la simplification des traitements ultérieurs des images; les points qui se trouvent en dehors de cet intervalle seront considérés comme ayant changé. formation entre TM1 (bleu), TM2 (vert), et TM3 (rouge) d'une part, TM5 et TM7 d'autre part (moyen infra-rouge tous les deux). Nous avons donc par la suite limité l'étude aux canaux TM3 (rouge), TM4 (proche infrarouge) et TM5 (moyen infra-rouge).

\section{Coefficients de normalisation}

La méthode de normalisation a été appliquée aux canaux TM3, TM4, TM5 (rouge, proche et moyen infra-rouge) des images Landsat de 88, 90 et 91 , prises 2 à 2. Dans le tableau I sont indiqués les coefficients a et $b$ calculés lors de la normalisation intermédiaire à partir des caractéristiques de l'ensemble de la zone forestière, les coefficients $a^{\prime}$ et $b^{\prime}$ calculés lors de la normalisation définitive par régression linéaire, ainsi que certains paramètres de la régression et les résultats du test utilisé pour savoir si la différence entre les 2 jeux de coefficients est significative. Ce test est le suivant :

On teste l'hypothèse $\mathrm{HO}:$ " $\mathrm{a}^{\prime}=\mathrm{a}$ et $\mathrm{b}^{\prime}=$ b " contre H1: " $a \neq a$ ou $b^{\prime} \neq b$ ".

Si $\mathrm{HO}$ est vraie, $\mathrm{F}=1 / 2 \sigma^{2}\left(\mathrm{n}\left(\mathrm{b}^{\prime}-\mathrm{b}\right)^{2}+\right.$ $\left.2 n \cdot \mu_{\mathrm{CN} 1}\left(a^{\prime}-a\right)\left(b^{\prime}-b\right)+\left(a^{\prime}-a\right)^{2} \cdot \Sigma C N_{1 i}{ }^{2}\right)$ est la réalisation d'une variable $F(2, n-2)$ (Saporta, 1990).

On s'aperçoit alors que :

- l'hypothèse de linéarité n'est pas aberrante; dans tous les cas les coefficients de détermination, compris entre 0,96 et 0,99 , sont hautement significatifs (testés par la variable $t=R \sqrt{ }(n-2) / \sqrt{1-R^{2}}$, valeur absolue d'une variable de Student à $n-2$ degrés de liberté) et l'étude de la répartition des résidus ne laisse rien apparaître de «suspect"; le modèle linéaire semble acceptable.

- dans certains cas les coefficients $a, b$ et $a^{\prime}, b^{\prime}$ sont significativement différents, ce qui justifie le calcul de $a^{\prime}$ et b' et semble montrer que les changements sont parfois assez importants pour qu'une normalisation simple 
Tableau I. Comparaison des coefficients de normalisation calculés à partir de $\mu_{f}$ et $\sigma_{f}$ et par régression linéaire.

\begin{tabular}{|c|c|c|c|c|c|c|c|c|c|}
\hline \multirow{2}{*}{$\begin{array}{l}\text { Année if }\left(^{*}\right) \\
\text { Canal }\end{array}$} & \multicolumn{3}{|c|}{$90 / 88$} & \multicolumn{3}{|c|}{$91 / 90$} & \multicolumn{3}{|c|}{$91 / 88$} \\
\hline & $T M 3$ & TM4 & TM5 & TM3 & TM4 & TM5 & TM3 & TM4 & TM5 \\
\hline \multicolumn{10}{|c|}{ Coefficients calculés à partir de $\mu_{f}$ of $\sigma_{f}$} \\
\hline a & 0,75 & 0,89 & 0,77 & 1,38 & 1,09 & 1,13 & 1,03 & 0,98 & 0,88 \\
\hline b & 11,10 & 28,05 & 22,07 & $-9,13$ & $-8,99$ & $-7,56$ & 4,25 & 20,02 & 16,22 \\
\hline \multicolumn{10}{|c|}{ Coefficients calculés par régression linéaire } \\
\hline$a^{\prime}$ & 0,72 & 0,88 & 0,83 & 1,41 & 1,07 & 1,11 & 0,99 & 0,96 & 0,96 \\
\hline$b^{\prime}$ & 11,57 & 28,29 & 19,30 & $-9,69$ & $-7,22$ & $-9,12$ & 4,94 & 20,45 & 12,36 \\
\hline \multicolumn{10}{|c|}{ Paramètres de la régression } \\
\hline Nombre de parcelles & 142 & 121 & 125 & 141 & 119 & 127 & 141 & 117 & 127 \\
\hline Coef détermination & 0,97 & 0,98 & 0,98 & 0,97 & 0,99 & 0,99 & 0,96 & 0,97 & 0,98 \\
\hline$\sigma$ estim de CN2n & 0,62 & 3,01 & 2,11 & 0,83 & 2,92 & 2,07 & 0,69 & 3,72 & 2,13 \\
\hline \multicolumn{10}{|c|}{ Test d'hypothèse sur l'égalité des coefficients } \\
\hline $\mathrm{F}$ & 8,53 & 2,91 & 23,03 & 1,90 & 1,71 & 2,75 & 3,46 & 2,07 & 26,90 \\
\hline Fseuil $(\alpha=0,05)$ & 3 & 3,07 & 3,07 & 3 & 3,07 & 3,07 & 3 & 3,07 & 3,07 \\
\hline Décision & \# & $=$ & \# & $=$ & $=$ & $=$ & \# & $=$ & $\#$ \\
\hline Fseuil $(\alpha=0,1)$ & 4,61 & 4,7 & 4,7 & 4,61 & 4,7 & 4,7 & 4,61 & 4,7 & 4,7 \\
\hline Décision & $\#$ & $=$ & $\#$ & $=$ & $=$ & $=$ & $=$ & $=$ & $\#$ \\
\hline
\end{tabular}

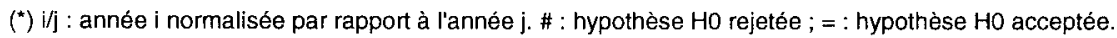

utilisant les coefficients $a$ et $b$ soit insuffisante.

L'influence du choix des parcelles a été en partie étudiée pour TM4 ; le calcul des coefficients de la droite de régression entre les données 90 et 91 avec un autre lot de parcelles a donné des résultats qui ne présentent pas une différence significative par rapport à ceux obtenus avec les premières parcelles.

\section{Seuils de changement}

Le tableau II indique le seuil $S$ de changement calculé pour 3 valeurs de $\mathrm{CN}_{2}: \mathrm{CN}_{2}=$ $\mu_{\mathrm{CN} 2}, O, 2 \mu_{\mathrm{CN} 2}$ où $\mu_{\mathrm{CN} 2}$ représente la moyenne des comptes numériques $\mathrm{CN} 2$ de l'ensemble des parcelles inchangées. Ces valeurs sont comparées à $\sigma$, erreur standard de l'estimation de CN2 normalisé.

Dans notre cas, avec un nombre suffisant d'observations ( $>50$ ), on peut approximer l'intervalle de confiance, limité par 2 branches d'hyperboles, par une région limitée par 2 droites parallèles à la droite de régression et distantes d'elle de $2 \sigma$. Cette approximation est d'autant plus justifiée qu'il n'est pas possible d'avoir une précision inférieure à l'unité au niveau des comptes numériques.

On peut alors calculer les différences, d'une part, entre les images 90 Normalisée et 88 , d'autre part, entre les images 91 Normalisée et 90 et comparer les valeurs obtenues aux seuils $S$ correspondants. 
Tableau II. Comparaison des valeurs moyennes et extrêmes de l'intervalle de confiance au seuil $\alpha=0,05$ à l'erreur standard de l'estimation de $\mathrm{CN}_{2 n}, \sigma$, donnée dans la table de régression $(t(n-2 ; \alpha / 2) \approx 1,980$ dans tous les cas considérés).

\begin{tabular}{|c|c|c|c|c|}
\hline \multirow[t]{2}{*}{ Année } & \multirow[t]{2}{*}{ Canal } & \multicolumn{2}{|c|}{ Seuil de différenciation $S$ calculé pour } & \multirow{2}{*}{$\begin{array}{c}S=2 \sigma \\
\sigma \text { erreur standard } \\
\text { de l'estimation de CN2n }\end{array}$} \\
\hline & & $\left(C N_{2}\right)_{0}=\mu_{C N 2}$ & $\left(\mathrm{CN}_{2}\right)_{0}=0$ ou $2 \mu_{\mathrm{CN} 2}$ & \\
\hline $90 / 88$ & TM3 & 1,23 & 1,28 & 1,24 \\
\hline $90 / 88$ & TM4 & 5,98 & 6,23 & 6,02 \\
\hline $90 / 88$ & TM5 & 4,19 & 4,34 & 4,22 \\
\hline $91 / 90$ & TM3 & 1,65 & 1,85 & 1,66 \\
\hline $91 / 90$ & TM4 & 5,8 & 6,11 & 5,84 \\
\hline $91 / 90$ & TM5 & 4,11 & 4,28 & 4,14 \\
\hline $91 / 88$ & TM3 & 1,37 & 1,53 & 1,38 \\
\hline $91 / 88$ & TM4 & 7,4 & 7,82 & 7,44 \\
\hline $91 / 88$ & TM5 & 4,23 & 4,40 & 4,26 \\
\hline
\end{tabular}

\section{Analyse des zones où une évolution radiométrique est détectée}

L'image obtenue directement par différence entre Ima2Norm et Ima1 suivie d'un seuillage est bruitée : au niveau des zones présentant une certaine texture et le long des limites, de nombreux pixels ont une valeur sur la différence d'images en dehors de l'intervalle $[-S$; + S]. Ceci s'explique par les légers décalages qui persistent entre images après correction géométrique. Le lissage à l'aide d'un filtre moyen des images Ima2Norm et Ima1 permet de supprimer ces artefacts pour les zones texturées.

Quarante-cinq sites pour lesquels une différence significative de radiométrie est détectée ont été visités ; 43 avaient évolué : coupes rases, incendies, éclaircies, dégagements, travaux sur chemins ou pistes...

Pour 2 sites seulement, l'évolution radiométrique n'a pu être expliquée par une intervention humaine ou un accident : I'un dans un taillis de feuillus, l'autre dans un jeune reboisement de résineux où le développement normal du peuplement pourrait être la cause du changement de radiométries.
La majorité des changements a été perçue sur TM4 (proche infra-rouge). Cependant les zones incendiées sont mieux détectées à partir de TM5 (moyen infra-rouge) ; avec TM4 seules les parties les plus touchées par le feu semblent être détectées et les surfaces incendiées sont sous-estimées.

Nous avons donc constaté que $95 \%$ des sites identifiés par traitement d'image et vérifiés sur le terrain avaient subi une transformation qui pouvait expliquer l'évolution radiométrique.

\section{Analyse des parcelles éclaircies ou coupées à blanc signalées par I'ONF}

Pour l'analyse de l'évolution radiométrique de ces parcelles, nous avons été gênés par des imprécisions à 2 niveaux :

- situation des interventions dans le temps: on connaît les dates de permis d'exploitation et de décharge d'exploitation concernant une parcelle, qui sont parfois distantes de 3 ou 4 ans. La date de la coupe est comprise entre ces 2 dates mais cette information 
n'est pas toujours suffisante pour situer la coupe par rapport aux prises de vues. Les données pour lesquelles on a pu situer l'intervention par rapport aux prises de vues sont alors les plus intéressantes ;

- localisation des coupes dans l'espace car, si on sait qu'une parcelle a été coupée en partie seulement, la localisation de cette coupe partielle est inconnue.

\section{Coupes rases}

Toutes les coupes rases répertoriées présentaient une évolution significative des radiométries, sur les canaux TM4 et TM5. Ces évolutions radiométriques sont liées soit à l'extraction de la matière végétale si la coupe a effectivement lieu entre les 2 prises de vues, soit aux changements importants du milieu après la coupe. En effet, des coupes réalisées avant 1988 ont été repérées ; en 1988 le milieu est alors en régénération, et c'est cette évolution que l'on perçoit.

\section{Éclaircies}

Les résultats concernant une partie des éclaircies sont rapportés dans les tableaux III et IV.

Le meilleur canal pour détecter les éclaircies est le proche infra-rouge (TM4). Ce résultat concorde avec ceux obtenus dans d'autres études où le proche infra-rouge utilisé seul ou combiné avec le rouge (Pilon et al, 1988 ; Häme, 1987 ; Sader et Winne, 1992 ; Abednego et Collet, 1992 ; Herwitz et al, 1990) permet une bonne estimation de la masse du couvert forestier et de ses évolutions.

Le nombre de coupes d'éclaircie détectées est élevé ; si on regarde les résultats du tableau III, qui sont les plus fiables, seulement 1 éclaircie sur 12 n'a pas été détectée et ce sûrement en raison de la nature de la parcelle. De même, pour les parcelles répertoriées dans le tableau IV, un grand nombre de celles pour lesquelles les éclaircies n'ont pas été détectées sont très hétérogènes (vérifié sur photographies aériennes). Sur

Tableau III. Résultats pour les éclaircies dont la date d'exploitation est bien située par rapport aux prises de vues.

\begin{tabular}{|c|c|c|c|c|}
\hline \multirow{2}{*}{$\begin{array}{l}\text { Dates des } \\
\text { éclaircies }\end{array}$} & \multirow{2}{*}{$\begin{array}{l}\text { Nombre total } \\
\text { d'éclaircies }\end{array}$} & \multicolumn{3}{|c|}{ Nombre d'éclaircies repérées sur } \\
\hline & & $\begin{array}{c}T M 3 \\
\text { (rouge) }\end{array}$ & $\begin{array}{r}T M 4 \\
(P I R)\end{array}$ & $\begin{array}{c}T M 5 \\
\text { (MIR) }\end{array}$ \\
\hline $\begin{array}{l}\text { Antérieures à } 88 \\
P<D<11\end{array}$ & 5 & 2 & 5 & 4 \\
\hline $\begin{array}{l}\text { Entre } 88 \text { et } 90 \\
11<P<D<12\end{array}$ & 7 & 2 & 6 * & 3 \\
\hline $\begin{array}{l}\text { Entre } 90 \text { et } 91 \\
12<P<D<13\end{array}$ & 0 & 0 & 0 & 0 \\
\hline
\end{tabular}

\footnotetext{
* L'éclaircie non détectée concerne une parcelle très hétérogène pour laquelle la coupe n'a pu affecter qu'une faible partie de la surface. $P$ = date de permis d'exploitation $; D=$ date de décharge d'exploitation $; 11=$ date de prise de vue de l'image $88 ; 12$ = date de prise de vue de l'image $90 ; 13=$ date de prise de vue de l'image 91 .
} 
Tableau IV. Résultats pour les éclaircies dont la date d'exploitation ne peut pas être située précisément par rapport aux prises de vues.

\begin{tabular}{lcccc}
$\begin{array}{l}\text { Dates des } \\
\text { éclaricies }\end{array}$ & $\begin{array}{l}\text { Nombre total } \\
\text { d'éclaircies }\end{array}$ & $\begin{array}{c}\text { TM3 } \\
\text { (rouge) }\end{array}$ & $\begin{array}{c}\text { Nombre d'éclaircies repérées sur } \\
\text { (PIR) }\end{array}$ & $\begin{array}{c}\text { TM5 } \\
\text { (MIR) }\end{array}$ \\
\hline $\begin{array}{l}\text { Antérieures à } 90 \\
P<11<D<12\end{array}$ & 15 & 3 & 11 & 6 \\
$\begin{array}{l}\text { Antérieures à } 91 \\
P<11<12<D<13\end{array}$ & 6 & 1 & 3 & 2 \\
$\begin{array}{l}\text { Entre } 88 \text { et } 91 \\
11<P<12<D<13\end{array}$ & 17 & 3 & 8 & 7 \\
\hline
\end{tabular}

$P=$ date de permis d'exploitation $; \mathrm{D}=$ date de décharge d'exploitation $; 11$ = date de prise de vue de l'image 88 ; $12=$ date de prise de vue de l'image $90 ; 13=$ date de prise de vue de l'image 91 .

ces parcelles, digitalisées en totalité, l'éclaircie n'a pu affecter qu'un petit pourcentage de la surface ; le changement radiométrique n'est plus perçu au niveau de la moyenne radiométrique de la parcelle. Cependant on ne sait pas si elles n'ont pas été repérées pour cette raison ou à cause de la date de coupe.

Des parcelles éclaircies avant 1988 présentent un changement de radiométrie significatif entre 1988 et 1990 . Ce résultat s'explique si la coupe a été réalisée peu de temps avant la prise de vue 1988, date à laquelle le peuplement ne s'est pas encore refermé. Dans ce cas là on observe une augmentation des comptes numériques sur TM4 entre les 2 dates au lieu de la diminution constatée lorsque l'éclaircie a lieu entre les 2 prises de vue.

Sur le canal TM5, l'évolution des comptes numériques n'est pas toujours expliquée par des interventions humaines sur les peuplements. Plusieurs études ont montré l'intérêt de ce canal pour détecter les peuplements malades ou dépérissants (Häme, 1987; Godard et al, 1990) mais les varia- tions observées dans le moyen infra-rouge sont souvent difficile à interpréter.

En ce qui concerne la "qualité» de l'éclaircie, on n'a pas pu mettre en évidence de relation entre l'importance de la différence radiométrique et l'intensité de la coupe. Ceci peut encore s'expliquer par le tracé des parcelles qui est souvent plus large que celui de l'éclaircie elle-même.

\section{Accidents}

Deux parcelles, exploitées après la prise de vue 1991, présentaient cependant une évolution radiométrique significative liée à la présence d'un chablis survenu avant cette date.

\section{DISCUSSION}

La méthode de comparaison d'images proposée est simple à mettre en œuvre car basée sur l'information contenue dans les images. Elle est adaptée aux milieux relativement stables d'une année à l'autre, ce 
qui est le cas de certains milieux forestiers, dont celui étudié. Pour l'instant les images doivent être prises dans les mêmes conditions d'observation, ce qui limite le choix des images à traiter. Dans le cas étudié, les images Landsat TM de 90 et 91 présentaient des conditions optimales d'application : jours de prise de vue quasiment identiques, une année d'intervalle, donc peu de changements. Mais il n'est pas toujours possible d'avoir des images de bonne qualité prises le même jour d'une année à l'autre. La différence de $20 \mathrm{j}$ environ entre les images de 88 et 90 et la différence de $6,6^{\circ}$ qui en résulte au niveau de l'angle d'incidence solaire ne semble pas avoir diminué le taux de détection des changements. Si I'hypothèse de linéarité s'est avérée acceptable dans des conditions d'observation similaires, il est peu probable qu'elle reste valable lorsque les angles d'incidence solaire ou de prise de vue vont varier de façon importante entre 2 dates. Les possibilités de généralisation et d'adaptation de cette méthode à des images prises avec des angles de visée différents (images SPOT) sont en cours d'étude. L'utilisation de données d'origines différentes permet, entre autres, d'augmenter les chances d'acquisition des images à la date souhaitée.

En raison des légers décalages qui persistent après les corrections géométriques, les images ont été lissées pour supprimer certains artefacts. Ainsi l'information texturale est en partie perdue et seules les évolutions radiométriques moyennes peuvent être perçues. Cependant, sur la zone étudiée, on a pu mettre en évidence des informations intéressant les professionnels forestiers: présence d'incendies, coupes rases, coupes d'éclaircies, chablis, quelques travaux de dégagement... II n'est pas étonnant de détecter des perturbations telles que les coupes rases à $100 \%$. II est en revanche intéressant de noter le bon taux de détection des éclaircies (11/12 pour celles dont la date d'exploitation était à peu près connue) qui peut cependant être lié ici aux pratiques sylvicoles. Le taux de détection obtenu pour les autres parcelles (date d'exploitation mal située par rapport aux prises de vue) est inférieur (22 éclaircies repérées sur 38) mais sûrement sous-estimé pour 2 raisons :

- si des parcelles ont été éclaircies longtemps avant la prise de vue de 1988 il est normal de ne pas les repérer ;

- mais surtout, bon nombre des interventions non détectées concernent des parcelles hétérogènes éclaircies partiellement. La radiométrie moyenne de la parcelle est peu modifiée mais la partie éclaircie pourrait apparaître lors de la cartographie.

Il faudrait maintenant augmenter le nombre de parcelles test pour confirmer les résultats obtenus.

II est possible que certains changements ou certains travaux sylvicoles n'affectent que la texture de l'image. Dans les images actuelles la résolution est insuffisante, même avant lissage, pour apporter une information texturale fine et la détection de tels changements est peu probable. Mais les futurs capteurs auront des résolutions plus fines et, dans une optique d'utilisation de leurs images, il faudra s'intéresser aux possibilités de suivi de l'évolution texturale, en plus de l'évolution radiométrique.

Pour l'instant nous n'avons pas abordé l'aspect «mise en forme cartographique» des informations extraites, mais simplement évalué la possibilité de détecter des changements en milieu forestier. Pour réaliser une carte il faudra prendre en compte les éventuels décalages résiduels entre images qui vont poser problème au niveau des limites entre peuplements de radiométries différentes. Une fois les changements bien localisés, l'utilisation croisée d'informations radiométriques et extérieures à l'image (en particulier celles de la carte des types de peuplements de I'IFN) devrait permettre l'identification de certains changements. 
Tous les changements forestiers susceptibles d'être repérés à partir d'images satellitaires ne conduisent pas à une modification du type de peuplement défini par I'IFN ; c'est le cas de la plupart des éclaircies. Il faudra donc accompagner la détection et lidentification des changements d'une réflexion sur la façon d'enregistrer cette information et de la présenter aux utilisateurs des données de l'IFN.

\section{REMERCIEMENTS}

Nos remerciements vont au CNES qui a financé le projet Spot \& Forêt 2 et l'acquisition des données Landsat pour l'étude de l'intérêt du moyen infrarouge, dans le cadre de la préparation du lancement de SPOT4.

Nous remercions M Nadal, du Pôle Télédétection, pour le prêt de certaines images. Merci au personnel de l'ONF de Castres pour son aide dans la récolte des informations de terrain et aux personnes de l'IFN qui nous ont permis de disposer des fichiers cartographiques et des photographies aériennes en temps voulu. Un grand merci à $A$ Vidal et $C$ Puech (laboratoire commun de télédétection ENGREF/CEMAGREF) pour l'aide apportée à la réalisation de cet article.

\section{RÉFÉRENCES}

Abednego BSP, Collet C (1992) A theoretical approach for vegetation change index based on non-corrected radiometric data.Int $J$ Remote Sens 13, 4, 699-714

Bazire $\mathrm{P}$ (1987) Télédétection et forêts. Rev For Fr2, 1987, 157-158

Deschamps PY, Herman M, Tanre D (1981) Influence de l'atmosphère en télédétection des ressources terrestres. Modélisation et possibilités de correction. Signatures spectrales d'objets en télédétection, Avignon, 811 sept 1981, 544-558

Deshayes M, Durrieu S, Girou D (1990) Utilisation de la télédétection satellitaire pour des applications forestières, projet SPOT \& Forêt 1 , rapport final, LCT ENGREF/CEMAGREF
Deshayes M, Durrieu S, Lauret S, Poupard C (1992) Projet SPOT \& Forêts 2, rapport intermédiaire, LCT ENGREF/CEMAGREF

Fontaine $A$ (1992) Utilisation des images satellites pour l'enrichissement et la mise à jour de cartes forestières, mémoire de fin d'études, ENGREF, Sept 1992, $72 \mathrm{p}$

Franklin J, Logan TL, Woodcock CE, Strahler AH (1986) Coniferous Forest Classification and Inventory Using Landsat and Digital Terrain Data, IEEE Trans Geosci Remote Sens, GE24, 1, janv 1986

Godard M, Gray J, Poitevin J (1990) The relative merits of SPOT HRV and Landsat TM images for forest cover change detection in Forillon National Park, Quebec, Canada, IEEE Trans Geosci Remote Sens 28, 4, 745-746

Gu XF (1988) Mise en relation des luminances mesurées par SPOT avec les réflectances de surfaces agricoles mesurées au sol, mémoire de DEA, université Paris VII, Juin $1988,37 p$

Häme T (1987) Satellite image-aided change detection. In: Proc SNS/Taksaattoriklubi, Seminar on Remote Sensing - Aided Forest Inventory (Ed University of Helsinki), 10-12 Dec, Research notes $\mathrm{n}^{\circ} 19$

Herwitz SR, Peterson DL, Eastman JR (1990) Thematic Mapper detection of changes in the leaf area of closed canopy pine plantations in Central Massachussetts. Rem Sens Environ $29,129-140$

IFN, Manuel du photointerprète du Tarn ( $3^{\mathrm{e}} \mathrm{cycle}$ )

Itten KI, Meyer P, Kellenberger T, Leu R, Sandmeier S, Bitter P, Seidel K (1992) Correction of the impact of topography and atmosphere on Landsat-TM forest mapping of alpine regions. Remote Sensing Series, vol 18, Department of Geography, University of Zurich, $48 \mathrm{p}$

Lauret S (1991) Télédétection et forêt : correction radiométrique des effets du relief, mémoire de fin d'études, INA-PG, $104 \mathrm{p}$

Leckie DG (1989) Innovation in remote sensing. In: Proc 13th Commonwealth Forestry Conference (GL Tarlton, ed), Rotorua, New Zealand, 17-30 Sept 1989, session 4A

Lepoutre D, Leprieur C, Peyron JL (1986) Télédétection et forêts : situation actuelle en Suède et perspectives pour la France. Rev For Fr XXXVIII-4, 385-393 
Lopez Garcia J (1987) Un metodo alternativo de correccion atmosferica, Universitat de Valencia

Peyron JL, Leprieur C, Durand JM (1988) Application de la télédétection à l'aménagement des forêts tempérées. Rev For Fr XL-n ${ }^{\circ}$ spécial, 1988, 85-90

Pilon PG, Howarth PJ, Bullock RA, Adeniyi PO (1988) An enhanced classification approach to change detection in semi-arid environments. Photogramm Eng Remote Sensing 54, 12, 1709-1716

Poupard C (1991) Normalisation d'images SPOT pour la cartographie forestière, mémoire de fin d'études, ENGREF, sept 1991, 104 p

Proy C, Leprieur C (1985) Influence de topographie et de l'atmosphère sur les mesures radiométriques en région montagneuse. Test d'un modèle d'inversion du signal sur des données TM, Proc 3rd Int Colloqium on Spectral Signatures of Objects in Remote Sensing, Les Arcs, France, 16-20 dec 1985, ESA SP-247
Richter R (1990) A fast atmospheric correction algorithm applied to Landsat TM images. Int $J$ Rem Sens 11, 1, 159-166

Sader SA, Winne JC (1992) RGB-NDVI colour composites for visualizing forest change dynamics. Int J Rem Sens 13, 3055-3067

Saporta G (1990) Probabilités, Analyse des données et Statistique, Éd Techniques, $493 p$

Strome WM, Leckie DG, Miller J, Buxton R (1991) Application of high resolution remote sensing image data. In: Proc Symp Amer Ins Aeronautics and Astronautics ( $\mathrm{F}$ Shahroki, ed) Washington, 77-93

USDA (1981) FOCIS: a forest classification and inventory system using Landsat and digital terrain data, United States Department of Agricultural Forest Service

Yang CJ, Vidal A (1990) Combination of digital elevation models with SPOT-1 HRV multispectral imagery for reflectance factor mapping. Remote Sens Environ 45, 35-45 